\title{
Phase-Driven Finite Element Model for Spatio-temporal Tracking in Cardiac Tagged MRI
}

\author{
Idith Haber $^{1}$, Ron Kikinis ${ }^{2}$, and Carl-Fredrik Westin ${ }^{2}$ \\ 1 Children's Hospital, Harvard Medical School, Boston MA, USA \\ 2 Brigham and Women's Hospital, Harvard Medical School, Boston MA, USA \\ idith.haber@tch.harvard.edu
}

\section{Introduction}

MRI tissue tagging [1] of the heart produces noninvasive markers within the muscle wall that can be used to measure motion and deformation (Fig. 19a). However, the widespread use of tissue tagging has been limited by time-consuming image post-processing. This paper outlines a method for automatic 3D tracking of LV motion from 2D MRI images acquired from multiple views. Since the tags form a repetitive pattern in the images, the local phase can serve as a material property that can be tracked. We first derive displacement from local image phase, a quantity previously used for estimating disparity between two 2D images in stereo vision [2, [8] or to measure relative deformation, or strain [7]. Displacement estimates have also been used for least square fitting of a 2D affine motion model to angiography images [5].

In the current work, we use phase-based displacement estimates to drive a 3D finite element deformable model of the left ventricle (LV). Our model enables reconstruction of 3D motion from 2D images and stabilization of the phase-derived displacement estimates, which are susceptible to noise and image artifacts. The basic framework for this model has been previously applied to 3D motion reconstruction of the right ventricles from user tracked tags [4, 3]. We here describe the geometry and constraints that enable phase-based displacement estimates to be used for automatically recovering 3D motion from tagged MR images.

\section{Methods}

1) Local Phase Estimation: Given an ideal sinusoidal signal, the phase is the argument of the analytic signal. For real signals, we use separable quadrature filters to estimate the analytic signal [6]. In a quadrature filter the real and imaginary parts form a Hilbert pair which makes the filter magnitude response phase invariant. For each coordinate direction $\mathbf{u}$ in the frequency domain, the filter is:

$$
F(\mathbf{u})=R(\rho) D(\hat{\mathbf{u}}),
$$

where $\rho$ is the magnitude and $\hat{\mathbf{u}}$ is the unit direction $\mathbf{u}$. The directional function $D(\hat{\mathbf{u}})$ is oriented along the initial direction of the tags. The radial frequency function $R(\rho)$ is a Gaussian function on a logarithmic scale, and defines the frequency characteristics of the quadrature filter. 

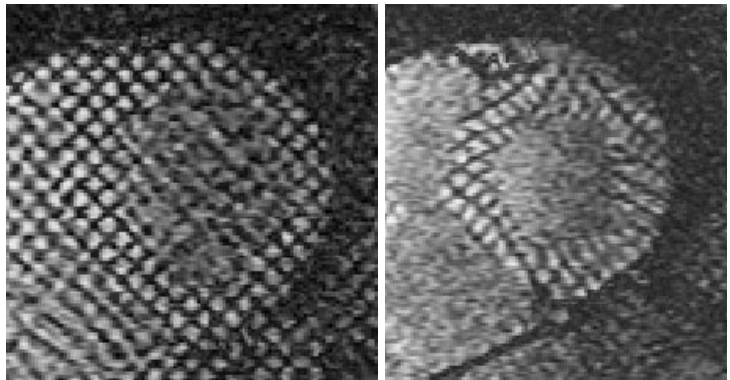

(a)

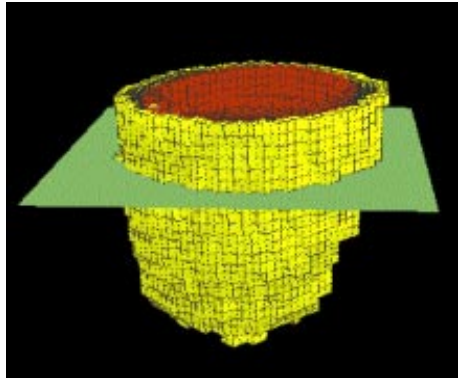

(b)

Fig. 1. (a) $2 \mathrm{D}$ tagged MR images of the left ventricle at beginning systole (relaxed) and end-systole (contracted). Dark bands represent tags that are tracked using local phase. (b) 3D finite element model used for simultaneously tracking in multiple images. A plane is drawn to show the location of the short-axis images.

In the spatial domain, we convolve the complex filter $\mathbf{f}$ with each pair of consecutive images $I_{t}, t=1,2$ to obtain $q_{t}=f(\mathbf{u}) * I_{t}$. The phase difference $\Delta \phi=\arg \left(q_{1} * q_{2}\right)$ is calculated with certainty $\left|q_{1} * q_{2}\right|$. We estimate the displacement to be $z=\frac{\Delta \phi}{2 \pi / \lambda}$, where $\lambda$ is the tag pattern wavelength. By applying quadrature filters to three mutually perpendicular tagging patterns, we can obtain a field of $3 \mathrm{D}$ displacement vectors, $\mathbf{z}$.

2) 3D FEM Deformable Model: Fitting a solid finite element model to the phase-based displacement data ensures smoothness and enables 3D motion estimation. The model geometry is defined using contours traced in the initial, end-diastolic images (Fig. 1b). The finite element formulation and equations governing the model dynamics are derived from the Lagrangian equations of motion [4, 9]:

$$
\dot{\mathbf{d}}=\mathbf{K d}+\mathbf{f}
$$

where $\mathbf{d}$ is the nodal displacement and $\mathbf{K}$ is the finite element stiffness matrix.

The external force $\mathbf{f}$ is a spring-like force designed to approximate the phase-derived displacement: $\mathbf{f}=\mathbf{z}-\mathbf{d}$. The model is iteratively fit between successive image frames using adaptive Euler integration until all forces are minimized.

\section{Results and Discussion}

Convolution of the quadrature filters with the images resulted in images of the spatial distribution of local phase (Fig. 2 a). These two images were then used to estimate the displacement, shown in Fig. 2b.

In order to assess the accuracy of the fitting, we applied the technique to synthetic image data. This data was generated by prescribing a complicated displacement field for a 3D superquadric geometry and calculating the intersection of this model with simulated image planes. We found that the difference between reconstructed and prescribed nodal displacement was less than one third of the voxel dimension. 


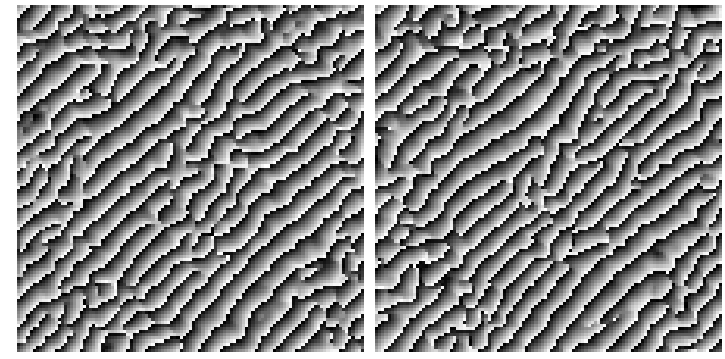

(a)
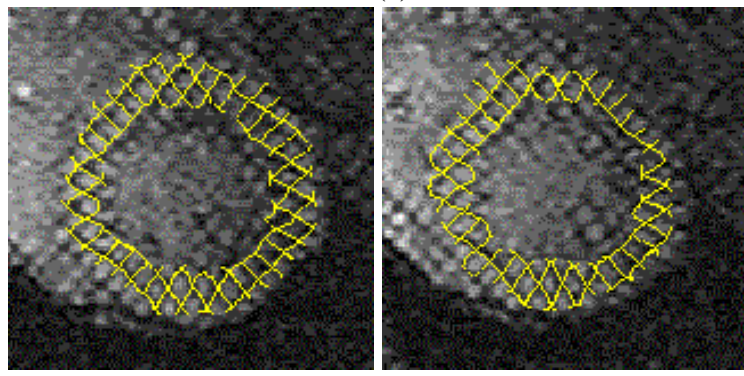

(c)

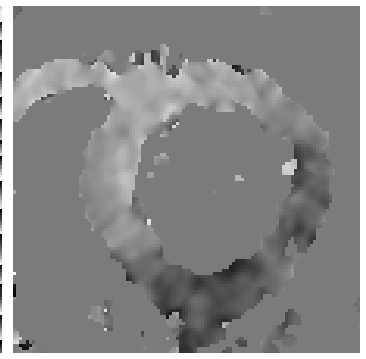

(b)

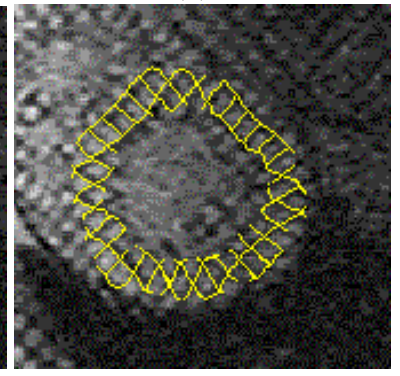

列

\section{(1)}

Fig. 2. Results: a) Phase calculated from consecutive images (Grayscale: $-2 \pi$ to $2 \pi$ ) b) Displacement derived from these two (Grayscale is $-2.5: 2.5 \mathrm{~mm}$ ). c) Representative results of fitting to image data from a normal volunteer. Intersection of model with image planes is shown along with images from consecutive short-axis slices.

The fitting technique was applied to images from several normal subject. We show a representative results Fig. 2 c. By viewing intersection points of the model with the original image planes are drawn over the original images, we can see a close agreement.

In conclusion, we describe a phase-based method for fitting a 3D model to tagged MRI. The model allows for the regularization of the phase estimates and integration of motion information from multiple image planes. We found good results when applying the method to MR images of normal hearts.

\section{Acknowledgements}

This work is funded in part by Boston Children's Heart Foundation, NIH HL63095 (IH), CIMIT and NIH P41-RR13218 (RK,CFW).

\section{References}

[1] L. Axel and L. Dougherty. Heart wall motion: Improved method of spatial modulation of magnetization for MR imaging. Radiology, 172(2):349-50, Aug 1989.

[2] D.J. Fleet, A.D. Jepson, and M. Jenkin. Phase-based disparity measurement. CVGIP: Image Understanding, 53(2):198-210, 1991. 
[3] I. Haber. Three-Dimensional Motion Reconstruction and Analysis of the Right Ventricle From Planar Tagged MRI. PhD thesis, University of Pennsylvania, Philadelphia, May 2000.

[4] I. Haber, D.N. Metaxas, and L. Axel. Three-dimensional motion reconstruction and analysis of the right ventricle using tagged MRI. Med Image Analysis, 4(4):335-355, 2000.

[5] M. Hemmendorff, H. Knutsson, M. T. Andersson, and T. Kronander. Motion compensated digital subraction angiography. In Proceedings of SPIE's International Symposium on Medical Imaging, volume 3661, San Diego, USA, February 1999. SPIE.

[6] H. Knutsson. Representing local structure using tensors. In 6th Scandinavian Conf. Image Analysis, pages 244-51, Oulu, Finland, June 1989.

[7] N. F. Osman, E.R. McVeigh, and J.L. Prince. Imaging heart motion using harmonic phase MRI. IEEE Trans on Med Imaging, 19(3):186-201, Mar 2000.

[8] T. Sanger. Stereo disparity computation using gabor filters. Biol Cybern, 59:405-418, 1988.

[9] O.C. Zienkiewicz and R.L. Taylor. The Finite Element Method. McGraw-Hill, New York, fourth edition, 1989. 